\title{
REVIEW
}

\section{Cost effectiveness of screening for Chlamydia trachomatis: a review of published studies}

\author{
E Honey, C Augood, A Templeton, I Russell, J Paavonen, P-A Mårdh, A Stary, \\ B Stray-Pedersen
}

See end of article for authors' affiliations

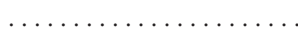

Correspondence to: Professor A Templeton, Department of Obstetrics and Gynaecology, University of Aberdeen, Aberdeen Maternity Hospital, Foresterhill, Aberdeen, AB25 2ZD, UK ; allan.templeton@abdn.ac.uk

Accepted for publication 16 August 2002
Objective: Screening for Chlamydia trachomatis in the lower genital tract may contribute to the prevention of pelvic inflammatory disease in women. The purpose of this review was to critically appraise, and summarise studies of the cost effectiveness of screening for $C$ trachomatis.

Methods: A literature search was conducted on Medline and in Health Star from 1990-2000. Keywords were $C$ trachomatis, screening, cost effectiveness. Bibliographies of reviewed articles were also searched. The population studied was asymptomatic sexually active women under 30 years of age in a primary care setting. The intervention assessed was screening for lower genital tract infection with $C$ trachomatis and the outcomes studied were cases of C trachomatis detected, cases of PID prevented, and associated costs. Studies were assessed using the Drummond criteria for economic evaluations. They were assessed qualitatively as they were too heterogeneous to allow quantitative analysis.

Results: 10 studies were included. All were modelled scenarios and all found screening to be more cost effective than simply testing symptomatic women, although all were based on probabilities that were assumed. Six of the studies focused on DNA based testing, three of them using urine. The models showed screening to be cost effective at prevalences of $3.1-10.0 \%$, and cost saving lovertesting symptomatic women) at a prevalence as low as $1.1 \%$, if age was used as a selection factor and DNA based tests were used in urine samples.

Conclusions: At the prevalence of infection expected in the target population, all studies suggest screening is cost effective. However, the assumptions used in the models have been difficult to confirm and there is a need for more data, particularly on the risk of complications in women with asymptomatic lower tract infection.
C hlamydia trachomatis is the most common, curable, bacterial sexually transmitted disease. ${ }^{1}$ It is a major cause of pelvic inflammatory disease (PID), which in turn is a major cause of infertility and ectopic pregnancy. ${ }^{2-5}$

The prevalence of lower tract infection with $C$ trachomatis varies from $2-25 \%{ }^{67}$ and is highest in young people. Infection with $C$ trachomatis places a considerable burden on health services throughout the developed world. ${ }^{8}$ The infection is asymptomatic in $75 \%$ of women and at least $50 \%$ of men, thus lower genital tract infection remains largely undetected.

Current screening practices differ throughout Europe and North America. For example, in Sweden there is an active detection policy targeting women under 25 , whereas in the United Kingdom there is no formal screening programme. ${ }^{10}$ Most of the evidence that supports screening for $C$ trachomatis as a way of preventing PID is derived from epidemiological data. $^{23111^{12}}$ The only randomised controlled trial (RCT) of screening for $C$ trachomatis was conducted in the United States ${ }^{13}$ and demonstrated that selective screening reduced the incidence of PID by $56 \%$ during 1 year of follow up.

A number of studies ${ }^{14-24}$ have assessed the cost effectiveness of screening for asymptomatic $C$ trachomatis. This review attempts to appraise these studies and to make recommendations for future practice and research.

\section{METHODS}

Articles addressing the cost effectiveness of screening for $C$ trachomatis were identified by a systematic computerised literature search and by searching the reference lists in relevant articles. An economic evaluation was considered eligible for inclusion if it assessed screening for $C$ trachomatis in primary care, defined as the first point of contact for healthcare provision, or family planning clinics (FPC) and the main focus was on sexually active women under the age of 30 . Studies examining men alone were excluded. The outcomes assessed were cases of PID prevented or cases of $C$ trachomatis detected.

The computerised literature search was performed in Medline, EMBASE, Health Star, and the NHS economic evaluation database (NEED). The MeSH key word "Chlamydia trachomatis" was expanded to "Chlamydia." The results of this search were combined with those of mass screening, economic evaluation, cost, cost benefit, and cost effective. The search was performed on literature from 1990-2000 because attitudes to screening and diagnostic technology have changed considerably in the past decade. Randomised controlled trials (RCT) of screening were also sought. The results of the search detected four studies that were not reported on NEED.

The following types of economic evaluations were nominated before assessment.

- A cost effectiveness analysis (including cost minimising), with a unidimensional outcome such as PID prevented

- A cost-utility analysis, where quality and quantity of life were examined-none of the studies located fell into this category

- A cost-benefit analysis, where benefits were valued and compared with costs and benefits of an alternative strategy.

Two reviewers (EH and CA) used the Drummond criteria ${ }^{25-28}$ to assess the methodology of the economic evaluations included in the systematic review (table 1). The criteria concur with the Task Force on Principles for Economic Analysis of Health Care Technology. ${ }^{29}$ The criteria are scored on the basis of a number of important issues including the identification of a focused and relevant question about a healthcare intervention, the level of 
Table 1 Drummond checklist for the appraisal of economic evaluations

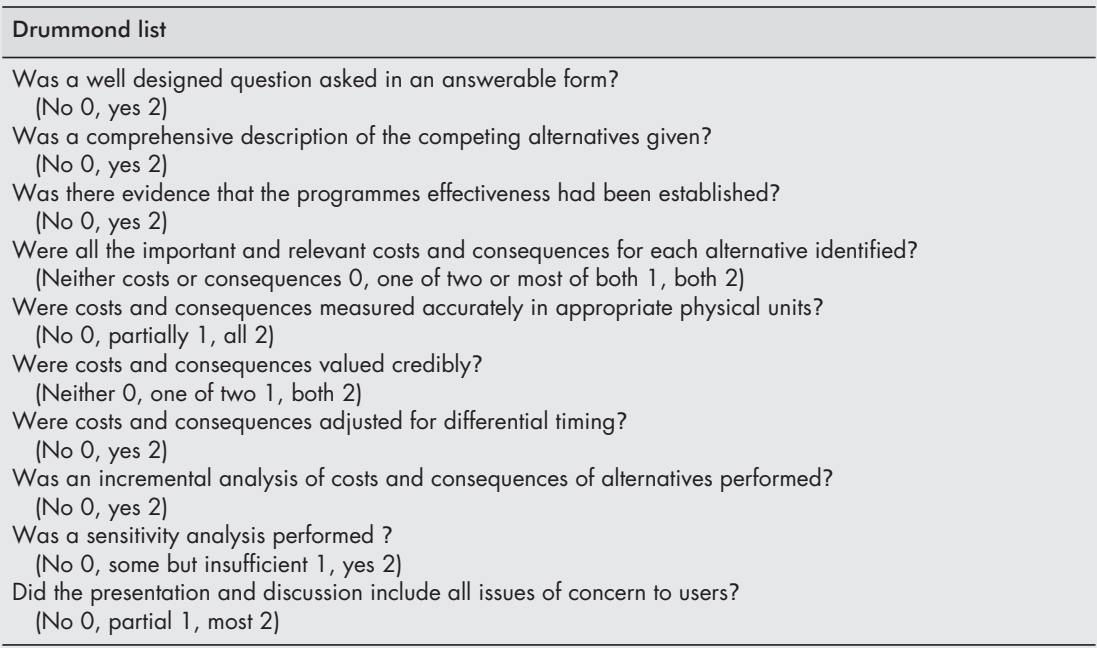

evidence used to measure the effectiveness of the intervention, and the validity of any assumptions in the evaluation. The use of a sensitivity analysis to assess the robustness of the evaluation and the generalisability of the findings are also scored. The scores attributed on the Drummond checklist were not weighted. Interobserver variability in the study assessment was quantified using kappa coefficient. Owing to heterogeneity no attempt was made to pool the results of the individual studies.

\section{RESULTS}

Ten economic evaluations and one RCT were identified. The $\mathrm{RCT}^{13}$ demonstrated that screening for $C$ trachomatis reduced the subsequent risk of PID, odds ratio 0.44 (0.2 to 0.9$)$. This RCT is the strongest available evidence that screening for $C$ trachomatis is effective and is described as grade I evidence. ${ }^{30}$ All other studies in this review were the result of economic modelling and not primary data collection. In five of the economic evaluations the prevention of PID was the measure of benefit assessed, while in the remainder $C$ trachomatis detected or cured was the measure. The agreement between the two reviewers ( $\mathrm{EH}$ and $\mathrm{CA}$ ) over the methodology of the economic evaluations had a kappa coefficient of $0.76(p<0.001)$. Three of the economic evaluations were by the same group of authors. Two of the studies ${ }^{1620}$ scored poorly (less than 5/20). Although they are included in table 2, they were excluded from further analysis.

In addition a further study was identified. ${ }^{31}$ This economic evaluation is set in the Netherlands and focuses on opportunistic screening of both men and women in general practice. The age range within the evaluation is $15-65$ years. These two factors meant it did not meet the inclusion criteria for the systematic review. It is none the less an interesting evaluation as it models the possible effect of screening this population for a period of 10 years, and demonstrates that a screening programme would have to run for a minimum of 5 years to create any savings.

The studies are summarised below.

Howell et $a^{24}$ asked which is the most cost effective strategy, screening women under 25 using LCR (ligase chain reaction) on urine, universally testing women or universally treating women? The effectiveness of these strategies was calculated hypothetically using data from a cohort study conducted in a similar population. The probabilities used for the consequences of untreated chlamydial infection were based on evidence from military clinic data and from a literature review.
The sensitivity of the test used was estimated from the literature. The costs used in this model reflect military health costs and loss of productivity to the military as well as the expense of losing trainees. The outcome measure was PID prevented at 1 year. In their model a hypothetical cohort of 10000 women with an infection prevalence of $9.2 \%$ was screened. They found that no screening would result in 276 cases of PID and the most effective strategy was screening only women under 25 which prevented 222 cases of PID and saved US\$15 (£9.7) for each case prevented. This is a reduction of $80 \%$ in PID and does not correlate with the Scholes study. This study was designed from a military perspective and as such the results may not apply generally.

Howell et al $^{22}$ asked if asymptomatic women under 30 in a family planning clinic (FPC) were to be screened, which would be the most cost effective test-cell culture, EIA (enzyme immunoassay), DNA probe, LCR, or PCR (polymerase chain reaction) either on urine or endocervical samples. The effectiveness of the strategies was based on a prevalence study conducted in the same population. The probabilities used in this review were derived from the literature and are based on cohort studies. The costs included are direct costs and the weighted costs of sequelae. The outcome measure was PID. The study was modelled on a cohort of 18000 women, with a prevalence of infection of $6.5 \%$. The cost if there was no screening was $\$ 2.2$ million ( $£ 1.42$ million) and 497 cases of PID; screening using EIA prevented 240 cases of PID and saved $\$ 887000$ ( $£ 571577$ ) over no screening. However, nucleic acid amplification based tests (NAA) used on urine prevented 306 cases of PID and saved an additional \$287000 (£184 935) over EIA. The use of a NAA based test remained the most cost effective method of screening with a prevalence as low as $3.2 \%$. The most cost effective scenario was a NAA based test used on a cervical swab in those women requiring a pelvic examination and on urine in those who did not.

Howell $e t a l^{23}$ asked if it was more cost effective to screen asymptomatic women in a FPC using the Centre for Disease Control and Prevention (CDC) criteria, or to screen all women under 30 or to universally screen all women when compared to no screening. The effectiveness of the strategies was determined as part of a cohort study set in the family planning clinics. Certain variables used in the model were derived from primary data collection at the time of screening-for example, physician time. The probabilities of consequences among women with untreated chlamydial infection were derived from a review of cohort studies in the literature. The costs used 


\begin{tabular}{|c|c|c|c|c|c|c|c|}
\hline & Author/date & Type of study & Test & Outcome & Case of PID prevented & Sensitivity analysis & $\begin{array}{l}\text { Score on } \\
\text { Drummond } \\
\text { criteria }(\max 20)\end{array}$ \\
\hline 1 & Howell et $a^{24}$ (1999) & $\begin{array}{l}\text { Model using real data from } \\
\text { records and previous cohort studies } \\
\text { Military oriented } \\
\text { High prevalence }(9.2 \%)\end{array}$ & LCR urine & $\begin{array}{l}\text { Age based testing or universal } \\
\text { treatment in high prevalence } \\
\text { groups }\end{array}$ & $\begin{array}{l}233 \text { cases of PID prevented saving } \\
\text { of } \$ 800 / \text { PID }\end{array}$ & $\begin{array}{l}\text { Yes, varied prevalence, cost, } \\
\text { uptake and sensitivity }\end{array}$ & 16 \\
\hline 2 & Howell et $a^{P^{3}}$ (1998) & $\begin{array}{l}\text { Model } \\
7 \text { strategies to decide on test } \\
\text { Extensive sequelae included } \\
\text { High prevalence }(9.2 \%)\end{array}$ & & $\begin{array}{l}\text { DNA amplification in urine or } \\
\text { cervical swab in asymptomatic } \\
\text { women less than } 30 \text { years. }\end{array}$ & $\begin{array}{l}306 \text { cases of PID prevented saving } \\
\$ 3689 / \mathrm{PID}\end{array}$ & $\begin{array}{l}\text { Yes varied prevalence, cost, uptake } \\
\text { and sensitivity }\end{array}$ & 17 \\
\hline 3 & Howell et $a^{P^{2}}$ (1998) & $\begin{array}{l}\text { Model } \\
\text { Cost effectiveness }\end{array}$ & Urine based NAA & $\begin{array}{l}\text { Age based screening at less than } \\
10.2 \% \text { prevalence. Universal } \\
\text { screening over } 10.2 \% \text { prevalence. } \\
\text { Both in FPC. }\end{array}$ & $\begin{array}{l}85 \text { cases of PID prevented saving } \\
\$ 3585 / \mathrm{PID}\end{array}$ & $\begin{array}{l}\text { Yes, varied prevalence, cost, } \\
\text { uptake and sensitivity }\end{array}$ & 17 \\
\hline 4 & Paavonen et a ${ }^{21}$ (1998) & $\begin{array}{l}\text { Model } \\
\text { Cost effectiveness }\end{array}$ & NAA on urine & $\begin{array}{l}\text { Cost effective in low prevalence } \\
(3.9 \%) \text { population using DNA } \\
\text { based test assuming } 90 \% \text { return } \\
\text { rate }\end{array}$ & $\begin{array}{l}50 \% \text { of sequelae prevented cost } \\
\$ 45 \text { per case of } \mathrm{C}^{*}\end{array}$ & Yes, extensive & 15 \\
\hline 5 & Marazzo et all ${ }^{19}$ (1997) & $\begin{array}{l}\text { Cross sectional study to provide } \\
\text { data }\end{array}$ & EIA in several settings & $\begin{array}{l}\text { Selective screening in GUM. } \\
\text { Universal screening in FPC. } \\
\text { Both with EIA. }\end{array}$ & $\begin{array}{l}44674 \text { cases of } C^{*} \text { prevented a } \\
\text { saving of } \$ 987 \text { per case } \\
47025 \text { cases prevented a saving } \\
\text { of } \$ 667\end{array}$ & Yes & 18 \\
\hline 6 & Genc and $\operatorname{Mardh}^{18}$ (1996) & $\begin{array}{l}\text { Model } \\
\text { Cost effectiveness } \\
\text { Using local data. } \\
\text { Dubious assumptions eg } 100 \% \\
\text { cures }\end{array}$ & NAA & $\begin{array}{l}\text { Cost effective in asymptomatic } \\
\text { women in FPC with DNA based test } \\
\text { at } 6 \% \text { prevalence, positives treated } \\
\text { with azithromycin. }\end{array}$ & NA & None was performed & 12 \\
\hline 7 & Sellors et al ${ }^{17}$ (1992) & $\begin{array}{l}\text { Model } \\
\text { Cost effectiveness } \\
\text { Data collected from longitudinal } \\
\text { study and selective criteria } \\
\text { developed }\end{array}$ & EIA & $\begin{array}{l}\text { Selective screening using set } \\
\text { criteria in low prevalence } \\
\text { populations. Effective using EIA. }\end{array}$ & NA & Yes & 17 \\
\hline 8 & Buhaug et al ${ }^{15}$ (1990) & $\begin{array}{l}\text { Model } \\
\text { Cost minimisation }\end{array}$ & Culture & $\begin{array}{l}\text { Cost effective in GP in women }<24 \\
\text { every } 2 \text { years }\end{array}$ & $\$ 1477$ per case of PID prevented & No & 11 \\
\hline Excluded & Cohen et a ${ }^{20}$ & $\begin{array}{l}\text { Prospective } \\
\text { Cost minimisation }\end{array}$ & NAA & $\begin{array}{l}\text { School based screening was cost } \\
\text { effective. }\end{array}$ & & & 3 \\
\hline Excluded & Humphreys et al'b & & & Universal screening in FPC & & & 5 \\
\hline
\end{tabular}


were direct costs and were calculated from the primary study. The costs of the sequelae were derived from other models. The women were tested with a NAA based test using an endocervical swab if a pelvic examination was indicated and urine if it was not. The outcome measure was PID prevented. They found screening all women under 30 would prevent the greatest number of cases of PID. This would still offer a saving over not screening even when the prevalence of infection was as low as $1.1 \%$. However it was not the most effective strategy as a large number of unnecessary tests are performed. In their sensitivity analysis if the prevalence increased from $6.6 \%$ to $10.0 \%$ then universal screening became the most cost saving strategy.

Paavonen $e t a^{21}$ asked if screening asymptomatic women using urine and NAA based tests in a FPC would be more cost beneficial than the current practice of not screening, but still testing symptomatic women with EIA and DFA confirmation on an endocervical sample. The effectiveness of the scenario was based on a pilot study of screening. The probability used for participation in the screening programme was based on the participation rate in the "cervical smear" screening programme in Finland. The diagnostic test performance was perhaps overestimated but this is explicitly stated. The probabilities of the consequences of chlamydial infection were determined from published cohort studies and expert opinion and are based on women who are culture positive for $C$ trachomatis. The costs included are direct costs only but these are explicitly stated, credible, and were discounted at $4 \%$ per year. The outcome measure was $C$ trachomatis detected. The cost per case detected was $\$ 46(£ 29.6)$ if participation was $75 \%$ compared to a cost of $\$ 50(£ 32.2)$ if no screening was performed. The net saving in the population, with a prevalence of C trachomatis infection of 3.5\%, was $\$ 3.5$ million ( $£ 2.25$ million). The threshold prevalence in this study was 3.9\% but savings increased with prevalence. This study had added value in that it examined the health benefits of $C$ trachomatis screening. Compared with no screening, screening and treating with the antibiotic azithromycin would result in $62 \%$ more cured patients and $50 \%$ fewer long term sequelae. This study is only in part generalisable as it is based heavily on the Finnish experience.

Marrazzo $\mathrm{et} \mathrm{al}^{19}$ examined the most cost effective strategyselective screening, universal screening, or testing only symptomatic women in FPC and in sexually transmitted disease (STD) clinics. The effectiveness of the strategies was estimated from a cross sectional survey of women in a FPC and in a STD clinic. The probabilities used were derived from a literature review and are as such based on the best available evidence. These probabilities are high however. For example they estimate that symptomatic PID will develop in up to $40 \%$ of women with untreated chlamydial infection and this is intuitively too high. The costs included are both direct and indirect and are explicitly stated and credible. The main outcome measure was untreated chlamydial infection. The selective screening criteria were age $<20$ years or any partner related risk. Selective screening detected $74 \%$ of infected women in FPC, when only $53 \%$ of women were screened. Universal screening apparently saved an additional \$667 (£430) per case prevented over selective screening. Universal screening detected $94 \%$ of cases in the STD clinic and required testing $77 \%$ of the clients. Selective screening was less costly than universal screening, saving $\$ 987$ ( $£ 636$ ) per case of $C$ trachomatis prevented as opposed to universal screening which cost $\$ 53$ (£34.2) per case prevented. They found that the universal screening was more cost effective when the prevalence was greater than $3.1 \%$ (range 2.1-3.5) in the FPC when EIA was used. In a sensitivity analysis it was demonstrated that if the probability of PID fell below 15\% following untreated cervicitis it became a dominant factor.

Genc and Mardh ${ }^{18}$ studied the best screening tool including culture, EIA with DFA confirmation or NAA based tests, when compared with no screening in asymptomatic women attend- ing FPC, youth clinics, or gynaecology clinics. The effectiveness of screening in these scenarios was based on probabilities derived from cohort studies. The sensitivities of all the tests were possibly overestimated and were not subject to a sensitivity analysis. The study included both direct and indirect costs but with only a brief description of the breakdown of those costs. The likelihood of complications in women who are infected is not given. Costs were subjected to discounting and this varied from $5-10 \%$. Although an explicit sensitivity analysis was not performed, randomly chosen probabilities were entered from the defined ranges. The outcome measure was cured $C$ trachomatis infection. The main finding was that with a prevalence of $6 \%$ or more screening with a NAA based test was the most cost effective strategy.

Sellors $e \mathrm{al}^{17}$ asked if it was better to screen young sexually active women in a FPC, universally or selectively (comparing two different selection strategies) using culture, EIA, or culture and EIA on endocervical samples. The strategies were derived retrospectively from a patient sample in a family planning clinic known to have $C$ trachomatis infection. The effectiveness of the strategies was calculated from a prospective cohort study performed in the student health clinic. The probabilities of outcomes of untreated chlamydial infections were derived from the literature. The costs used are the direct costs involved in the collection, analysis and treatment of screening and are explicitly stated. Furthermore the costs associated with the sequelae are not explained. The sequelae are estimated to occur 8 years in the future and the costs are discounted by $5 \%$ per year. A sensitivity analysis was conducted over the costs and probabilities of the consequences of chlamydial infection, on the performance of the selective criteria and the threshold prevalence was calculated for each scenario. The outcome examined was $C$ trachomatis detected. This was a cost minimising evaluation concentrating on costs saved rather than cases of $C$ trachomatis detected. The selective criteria were cervicitis, intermenstrual bleeding, discharge, or urinary frequency. Using these criteria they tested $55.3 \%$ of women and detected $83.3 \%$ of cases. On the basis of a new sex partner in the previous year $75.4 \%$ of women were tested and 93.3\% of cases detected. They demonstrated that, while costing less, selective screening detected less cases of $C$ trachomatis. This conclusion may in part be influenced by the choice of EIA as a test.

Buhaug et $\mathrm{al}^{15}$ asked if it was cost effective to screen asymptomatic young women for $C$ trachomatis in general practice using culture of endocervical samples when compared with no screening. The effectiveness of the strategy was calculated from one follow up study. The consequences and probability of the consequences of untreated chlamydial infections were estimated from the published literature. It is striking in this study that there was the assumption that $95 \%$ of those with a positive test would come forward for treatment and this would be successful in $90 \%$. The costs in this model include direct and indirect costs and seem appropriate by today's standards but are perhaps high for 1990. The main outcome measure was PID prevented. The authors also modelled the effect of screening on anticipated PID and ectopic pregnancies. They found screening to be cost effective among 18-24 year olds with a prevalence of infection of $8.4 \%$ (range $3.5-13.4 \%$ ). This review had added interest as the authors varied the prevalence and the consequences of infection, with age. It is also interesting, that even using culture, the most expensive method for the detection of $C$ trachomatis, screening was still cost effective.

Thus the method used to detect $C$ trachomatis varied among the models. Six used an NAA based detection system, half of these in urine. Although now proposed as the gold standard, ${ }^{32}$ NAA testing is still not routinely offered in all settings. This review indicates that while nucleic acid amplification based tests can cost more than alternative tests their greater sensitivity makes their use more cost effective. In a screening scenario the relatively high false negative rates using non-NAA based tests may limit any longer term savings. 
Table 3 Probabilities of consequences of lower tract infection used in the economic evaluations

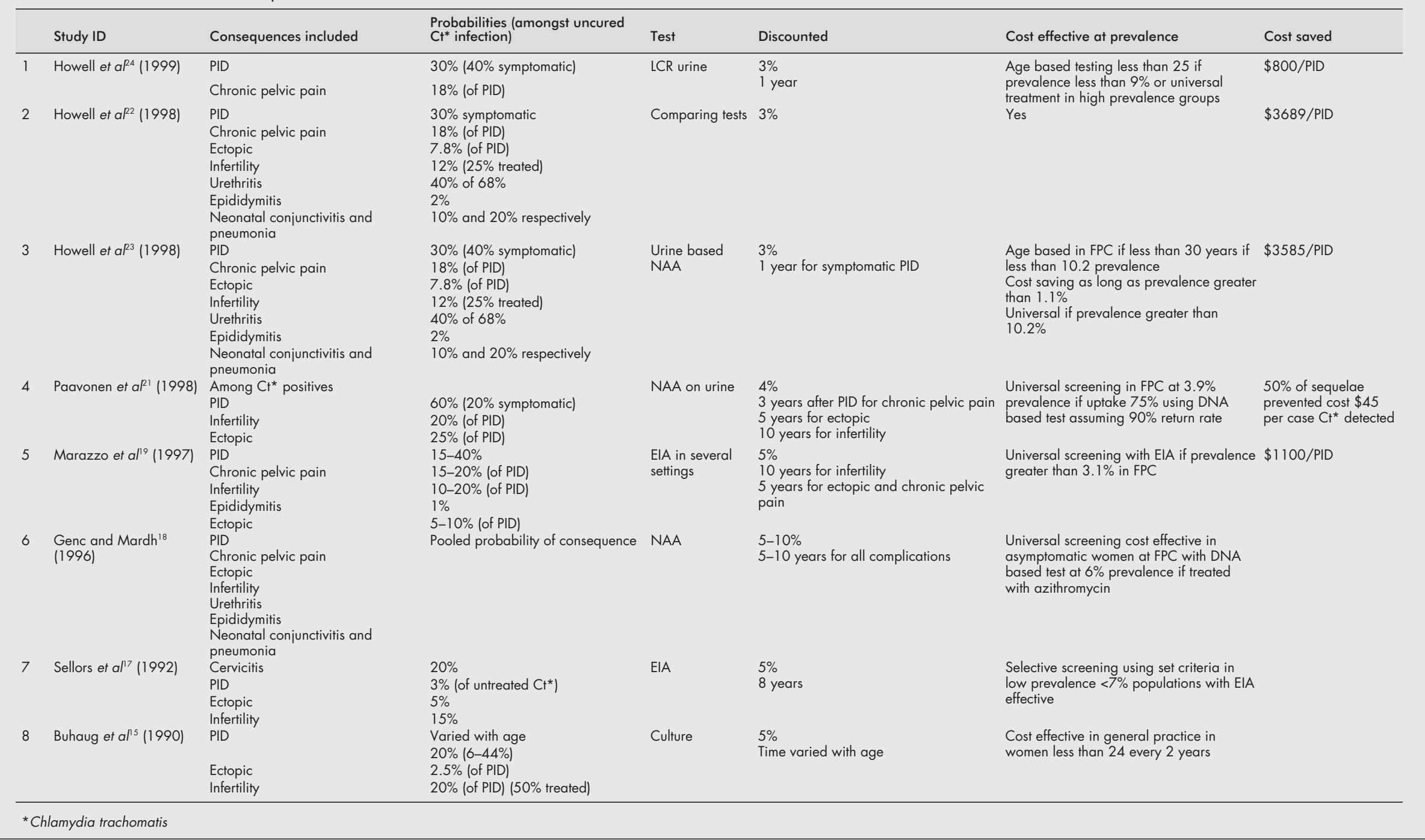


The threshold population prevalence of $C$ trachomatis over which the evaluations were cost effective varied from $3.1-10.0 \%$. Screening can be cost saving at a prevalence as low as $1.1 \%$, when age is used to select women and NAA based tests are used in urine samples, although the large numbers of unnecessary tests diminishes effectiveness. This variation in the threshold prevalence rate is explained by the very wide range of costs used in the models. ${ }^{33}$ It may also be explained by the decision in three evaluations not to include indirect costs. These costs, which are incurred by society and individuals, are important, although there is still much debate about their inclusion in an economic evaluation..$^{33}$ In two studies azithromycin in a single dose was used to treat the infection. ${ }^{18}{ }^{21}$ These studies had a threshold prevalence of $3.9 \%$ and $6.0 \%$ respectively. In the remainder doxycycline for 7 days was the standard treatment used. Both regimens have similar effectiveness but compliance is better with azithromycin. The probabilities of return for treatment and treatment failure ranged from $65-90 \%$ and $75-97 \%$ respectively, but at all these levels screening was still cost effective.

Incremental analysis was performed in six studies, demonstrating how much more one strategy cost over the next most effective strategy.

\section{DISCUSSION}

In this systematic review all the economic evaluations assumed de facto that screening for $C$ trachomatis was effective and then went on to agree that screening asymptomatic women for $C$ trachomatis is cost effective and indeed cost minimising over whatever scenario is currently offered. Medically this finding is unsurprising. The presumed consequences of lower genital tract infection with $C$ trachomatis are PID, ectopic pregnancy, and infertility-all of which can be very expensive in terms of both healthcare costs and lost productivity. ${ }^{34}$

Economic modelling can provide quick, accurate, and robust answers where an exploration of potential outcomes can be undertaken using a sensitivity analysis. However, this is dependent on the probabilities entered being substantiated by strong evidence. All of the models appraised in this review suffered from a lack of strong evidence to support their assumptions and are vulnerable therefore to bias in their findings and conclusions.

For example, the models have used probabilities and assumptions for the consequences of untreated $C$ trachomatis infection, which have been derived from a group of women who were culture positive for $C$ trachomatis. The assumption is that being culture positive is the same as being positive in a NAA based test, which is unlikely in relation to bacterial load alone. None of the models have used primary data from a cohort of women who have been screened for $C$ trachomatis using a sensitive diagnostic test and then followed up over time. None of the models including those published later used data derived from the Scholes study, ${ }^{13}$ the strongest evidence to date that screening is effective in preventing PID.

Although the models did conduct sensitivity analysis of prevalence rates, age groups, and types of screening, in only one of the studies did the researchers vary the uptake of screening. ${ }^{21}$ The other analyses were based on the presumption that the whole cohort accepted screening. However in the Scholes study, screening was accepted by only $64 \%{ }^{13}$ and a similar finding was noted by Grun et al. ${ }^{35}$ It is of course possible that uptake may improve with the advent of urine based testing as well as self testing using vulval swabs. Clearly there is a need for much more data if the cost effectiveness of screening is to be successfully modelled. These data would best be provided in the context of well conducted clinical trials.

There is now evidence, albeit from only one RCT, that screening for $C$ trachomatis is effective. ${ }^{13}$ One case of PID will be prevented for every 83 women screened; furthermore screening has been shown to reduce the rate of ectopic pregnancy within 1 year of introduction. ${ }^{12}$ However it is difficult to be certain that screening for $C$ trachomatis has been solely responsible for the fall in ectopic pregnancies or for the reduction in women with PID seen in Sweden, ${ }^{36}$ as screening interventions will have both a primary and secondary preventive action. Any national screening programme will increase awareness among the people at risk as well as healthcare professionals and this alone may bring about change.

\section{CONCLUSION}

This systematic review of the cost effectiveness of screening young asymptomatic women for Chlamydia trachomatis suggests that screening is cost effective at the prevalence of chlamydial infection expected in the relevant population. However, the assumptions upon which the models have been based are not always derived from sound evidence, particularly with respect to the subsequent risk of PID in women testing positive using newer sensitive nucleic acid based diagnostic tests. Further unanswered questions relate to the prevalence and reinfection rates of $C$ trachomatis after a screening programme has been established. There is an urgent need for more data derived from well conducted clinical trials to inform the discussion and help with national policies and recommendations.

\section{ACKNOWLEDGEMENT}

Project supported by EU Biomed Concerted Action Grant No: BMH4-98 3059.

Authors' affiliations

E Honey, A Templeton, Department of Obstetrics and Gynaecology, University of Aberdeen, Foresterhill, Aberdeen, AB25 2ZD, UK C Augood, Department of Epidemiology and Population Sciences, London School of Hygiene and Tropical Medicine, London, WC1E 7HT, UK

I Russell, Department of Health Sciences, University of York, Heslington, York, YOl 5DD, UK

J Paavonen, Department of Obstetrics and Gynecology, University of Helsinki, 00290, Helsinki, Finland

P-A Mårdh, Department of Obstetrics and Gynecology, University Hospital, 22285, Lund, Sweden

A Stary, Out-patient Center, Franz Jonas-Platz, A-1210 Vienna, Austria B Stray-Pedersen, Department of Obstetrics and Gynecology, National Hospital, N-0027, Oslo, Norway

\section{REFERENCS}

1 Taylor-Robinson D. Chlamydia trachomatis and sexually transmitted disease. BM 1994;308:150-1.

2 Cates WJ, Wasserheit JN. Genital chlamydial infections:epidemiology and reproductive sequelae. Am J Obstet Gynecol 1991;164:1771-81.

3 Svensson L, Mardh PA, Westrom L. Infertility after acute salpingitis with special reference to Chlamydia trachomatis. Fertil Steril 1983;40:322-39.

4 Westrom L. Incidence, prevalence, and trends of acute pelvic inflammatory disease and its consequences in industrialized countries. Am J Obstet Gynecol 1980;138:880-92.

5 Department of Health. Why mothers die. Report on the Confidential Enquiries into Maternal Deaths in the United Kingdom 1994-1996. London: HMSO, 1998

6 Svensson LO, Mares I, Mardh P-A, et al. Screening voided urine for Chlamydia trachomatis in asymptomatic adolescent females. Acta Obstet Gynecol Scand 1994;73:63-66

7 Tchoudomirova K, Nuhova P, Tchapanova A. Prevalence, epidemiological and clinical correlates of genital Chlamydia trachomatis infection. J Eur Acad Dermatol Venereol 1998;11:214-20.

8 Ripa T. Epidemiologic control of genital Chlamydia trachomatis infections. Scand J Infect Dis Suppl 1990;69:157-67.

9 Gaydos CA, Howell MR, Pare, B et al. Chlamydia trachomatis infections in female military recruits. N Engl J Med 1998;339:739-44.

10 CMO. Expert Advisory Group. Chlamydia trachomatis-summary and conclusions of Chief Medical Officer's Group. London: Department of Health, 1998: 1-22

11 Paavonen J, Lehtinen M. Chlamydial pelvic inflammatory disease. Human Reproduction Update 1996;2:519-29.

12 Egger M, Low N, Smith GD, et al. Screening for chlamydial infections and the risk of ectopic pregnancy in a county in Sweden: ecological analysis. BM 1998;316:1776-80. 
13 Scholes D, Stergachis A, Heidrich FE, et al. Prevention of pelvic inflammatory disease by screening for cervical chlamydial infection. $N$ Engl J Med 1996;334:1362-6.

14 Donaldson C, Shackley P. Economic evaluation. In: Detels R, Holland W, McEwan J, Omenn F, eds. Oxford textbook of public health. 3rd ed. Oxford: Oxford University Press, 1995;26:849-71.

15 Buhaug $\mathbf{H}$, Skjeldestad FE, Halvorsen LE, et al. Should asymptomatic patients be tested for Chlamydia trachomatis in general practice? $\mathrm{Br} J$ Gen Pract 1990:40:142-5

16 Humphreys JT, Henneberry JF, Rickard RS, et al. Cost-benefit analysis of selective screening criteria for Chlamydia trachomatis infection in women attending Colorado family planning clinics. Sex Transm Dis 1992;19:47-53.

17 Sellors JW, Pickard L, Gafni A, et al. Effectiveness and efficiency of selective vs universal screening for chlamydial infection in sexually active young women. Arch Intern Med 1992;152:1837-44.

18 Genc M, Mardh AA. A cost-effectiveness analysis of screening and treatment for Chlamydia trachomatis infection in asymptomatic women. Ann Intern Med 1996;124:1-7.

19 Marrazzo JM, Celum CL, Hillis SD, et al. Performance and cost-effectiveness of selective screening criteria for Chlamydia trachomatis infection in women. Implications for a national Chlamydia control strategy. Sex Transm Dis 1997:24:131-41.

20 Cohen DA, Nsuami M, Etame RB, et al. A school-based Chlamydia control program using DNA amplification technology. Pediatrics 1998;101:E1.

21 Paavonen J, Puolakkainen M, Paukku $M$, et al. Cost-benefit analysis of first-void urine Chlamydia trachomatis screening program. Obstet Gynecol 1998;92:292-8.

22 Howell MR, Quinn TC, Brathwaite W, et al. Screening women for chlamydia trachomatis in family planning clinics:the cost-effectiveness of DNA amplification assays. Sex Transm Dis 1998;25:108-17.

23 Howell MR, Quinn TC, Gaydos,CA. Screening for Chlamydia trachomatis in asymptomatic women attending family planning clinics. A cost-effectiveness analysis of three strategies. Ann Intern Med 1998; 128:277-84
24 Howell, MR, Gaydos, JC, McKee KTJ, et al. Control of Chlamydia trachomatis infections in female army recruits:cost-effective screening and treatment in training cohorts to prevent pelvic inflammatory disease. Sex Transm Dis 1999;26:519-26.

25 Drummond $M$, Stoddart $G$, Torrance $G$. Critical assessment of economic evaluation. In: Drummond M Stoddart $G$, Torrance $G$, eds. Methods for the economic evaluation of health care programmes. $1 \mathrm{st}$ ed. Methods for the economic evaluation of health care

26 Greenhalgh T. How to read a paper. Papers that tell you what things cost (economic analyses). BM 1997;315:596-9.

27 Udvarhelyi IS. Cost-effectiveness and cost-benefit analyses in the medical literature. Are the methods being used correctly? Ann Intern Med 1992; 1 16:238-44

28 Keenan SP, Massel D, Inman KJ, et al. A systematic review of the cost-effectiveness of noncardiac transitional care units. Chest 1998;113:172-7

29 Economic analysis of health care technology. A report on principles. Task Force on Principles for Economic Analysis of Health Care Technology. Ann Intern Med 1995;123:61-70.

30 Grimes DA, Atkins D. The US Preventative Task Force: putting evidence based medicine to work. Clin Obstet Gynecol 1998;41:332-42.

31 Welte R, Kretzschmar $M$, Leidl R, et al. Cost effectiveness for screening programmes for Chlamydia trachomatis. Sex Transm Dis 2000;27:518-29.

32 Lee HH, Chernesky MA, Schachter J, et al. Diagnosis of Chlamydia trachomatis genitourinary infection in women by ligase chain reaction assay of urine. Lancet 1995;345:213-16.

33 Mclntosh E, Donaldson C, Ryan M. Recent advances in the methods of cost-benefit analysis in healthcare matching the art to the science. Pharmacoeconomics 1999;15:357-67.

34 Stephenson JM. Screening for genital chlamydial infection. Br Med Bull 1998;54:891-902.

35 Grun L, Tassano-Smith J, Carder C, et al. Comparison of two methods of screening for genital chlamydial infection in women attending in general practice:cross sectional survey. BM 1997;315:226-30.

36 Kamwendo F, Forslin L, Bodin L, et al. Programmes to reduce pelvic inflammatory disease - the Swedish experience. Lancet 1998;351:25-8.

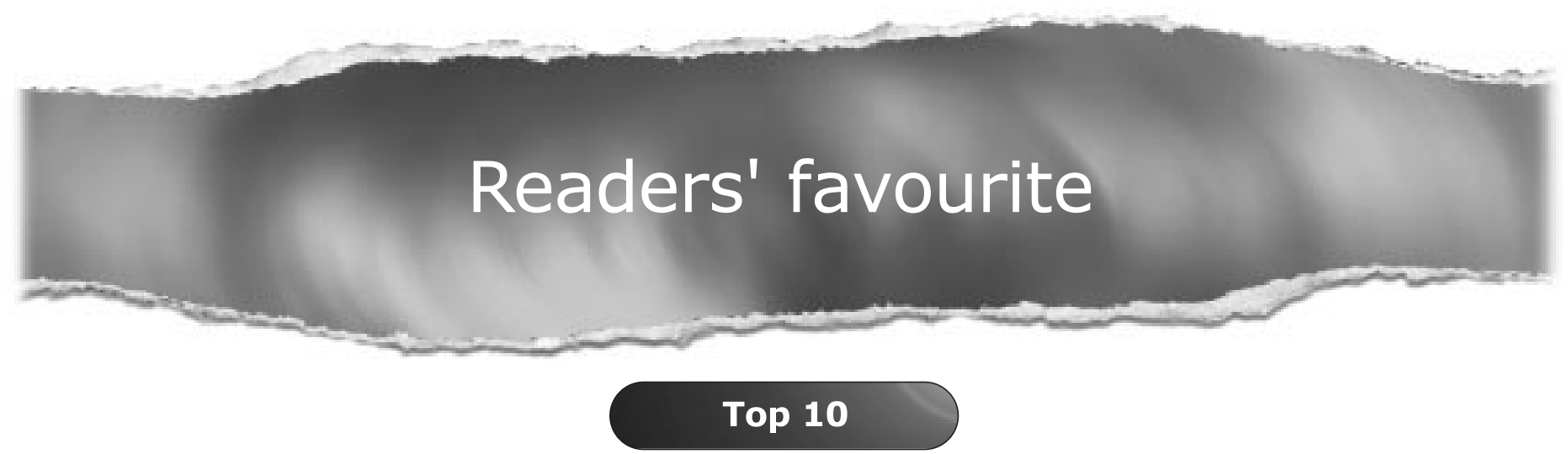

Click on the "Top 10" button on the homepage to see which are the best read articles each month

\section{www.sextransinf.com}

